\title{
Associating Lattice Fluids near Hard and Interacting Walls
}

\author{
R. H. C. J anssen and E. Nies* \\ Department of Polymer Technology, Eindhoven University of Technology, P.O. Box 513, \\ 5600 MB Eindhoven, The Netherlands \\ B. Smit \\ Shell Research and Technology Centre, P.O. Box 38000, \\ 1030 BN Amsterdam, The Netherlands
}

Received J une 17, 1996. In Final Form: September 4, 1996

\begin{abstract}
Cubiclatticemodels of dimerizing and polymerizing fluids havebeen studied within Wertheim's OrnsteinZernike(OZ) matrix formalism to assess theadsorption behavior of such fluids at impenetrable, interacting walls. The OZ matrix formalism is relatively easy-to-handle for these cubic lattice models, due to the absence of liquid-like packing effects. The calculated concentration profiles have been compared to NVTMonteCarlosimulations. The simulation of the polymerizing fluid employed a biased cluster move, based on the configurational bias scheme of Siepmann and Frenkel. The dimerizing fluid profiles are in nearly quantitative agreement with the simulation results, even for a fully associated, i.e., dimeric fluid. This is in contrast with the polymerizing fluid profiles that show important deviations from the simulation results, especially near interacting walls. It is ther efore concluded that better approximations are needed in solving Wertheim's OZ matrix for the polymerizing fluid.
\end{abstract}

\section{Introduction}

An associating fluid is characterized by short-ranged directional attractions between its particles. Studies of the bulk properties of associating fluids include the classical chemical theory of solutions ${ }^{1}$ in which the associativeinteractions aremodel ed as chemical reactions, lattice theories in which the free energy is minimalized within the quasi-chemical approximation, 2,3 computer simulation, and integral equation approaches (see ref 4 and references cited therein). The integral equation approaches attempt to accuratel y cal culatethestructural correlations between thefluid particles. Themacroscopic thermodynamic properties can then be calculated from these correlations. ${ }^{5}$

In this paper Wertheim's Ornstein-Zernike(OZ) matrix integral equation for associating fluids with one $e^{6,7}$ and two $0^{8,9}$ directional attractive sites per particle is adapted to study the adsorption of associating cubic lattice fluids at an impenetrable interacting wall. As far as we are aware, it is the first application of Wertheim's OZ matrix integral equations to obtain the adsorption profiles of associating lattice fluids. Recently, work that uses a different integral equation approach for continuum adsorbing fluids has also been reported. ${ }^{10}$

We study the adsorption of lattice fluids, instead of the more realistic continuum fluids, because the $\mathrm{OZ}$ matrix

\footnotetext{
* Author to whom correspondence should be addressed.

${ }^{\otimes}$ Abstract published in Advance ACS Abstracts, November 15 , 1996.

(1) Prausnitz, J . M.; Lichtenthaler, R. N.; Gomez de Azevedo, E. Molecular Thermodynamics of Fluid Phase Equilibria, 2nd ed.; Prentice-Hall: Englewood Cliffs, NJ, 1986.

(2) Guggenheim, E. A. Mixtures; Clarendon Press: Oxford, 1952.

(3) Besseling, N. A. M. Statistical Thermodynamics of Fluids with Orientation-Dependent Interactions. Ph.D. Thesis, Wageningen Agricultural University, Wageningen, The Netherlands, 1993.

(4) Henderson, D.; Sokolowski, S.; Pizio, O. J . Chem. Phys. 1995, $102,9048$.

(5) Hansen, J . P.; McDonald, I. R. Theory of SimpleLiquids, 2nd ed.; Academic Press: London, 1986.

(6) Wertheim, M. S. J. Stat. Phys. 1984, 35, 19.

(7) Wertheim, M. S. J . Stat. Phys. 1984, 35, 35.

(8) Wertheim, M. S. J . Stat. Phys. 1986, 42, 459.

(9) Wertheim, M. S. J . Stat. Phys. 1986, 42, 477

(10) Trokhymchuk, A.; Pizio, O.; Sokol owski, S.; Henderson, D. Mol

Phys. 1995, 86, 53.
}

formalism is very easy to handle in the case of lattice fluids. This is due to the absence of liquid-like ordering effects in such fluids. Also, it is very easy to introduce a flat wall into a cubiclatticefluid. ${ }^{11}$ Despitethesimplicity of thecubiclatticemodel, it retains themain characteristic of an associating fluid: the formation of orientationdependent bonds between thefluid particles. In thework reported here, wemainly consi der theadsorption of fluids for which theassociativeinteraction between the particles forms semi permanent (covalent) bonds that are not easily broken by thethermal motion of the particles. The fluids of particles with one (section 2) and two (section 3) directional attractive sites then serve to model the adsorption of molecular lattice fluids. Especially the doubly associating fluid is interesting to us, polymer chemists, for it models the adsorption of a polydisperse polymeric lattice fluid. Such polymeric lattice fluids are very commonly studied in polymer science ${ }^{12-16}$ and are technol ogically relevant in, e.g., thecompositeand coatings industries.

Theadsorption profiles that were obtained with the $\mathrm{OZ}$ matrix formal ism have been compared to Monte Carlo simulations. For thefluid with onedirectional attractive force per particle, the profiles were simulated by the standard brute force method. ${ }^{17}$ The doubly associating fluid was simulated with a biased cluster move ${ }^{18}$ which combines the configurational bias scheme $\mathrm{e}^{19}$ and a cluster move of Wu, Chandler, and Smit. ${ }^{20}$

The outline of the rest of this paper is as follows. In section 2 we delineate the equations for the calculation

(11) Henderson, D.; Abraham, F. F.; Barker, J . A. Mol. Phys. 1976, 31, 1291 .

(12) Scheutjens, J . M. H. M.; Fleer, G. J . J . Phys. Chem. 1979, 83, 1619.

(13) Helfand, E. Macromolecules 1976, 9, 307.

(14) Helfand, E. J . Chem. Phys. 1975, 63, 2192.

(15) Theodorou, D. N. Macromolecules 1988, 21, 1400.

(16) Fleer, G. J .; Cohen Stuart, M. A.; Scheutjens, J. M. H. M.; Cosgrove, T.; Vincent, B. Polymers at Interfaces; Chapman and Hall: London, 1993.

(17) Metropolis, N.; Rosenbluth, A. W.; Rosenbluth, M. N.; Teller, A. H.; Teller, E. J . Chem. Phys. 1953, 21, 1087.

(18) J anssen, R. H. C.; Nies, E.; Smith, B. Mol. Phys., submitted.

(19) Siepmann, J . I.; Frenkel, D. Mol. Phys. 1992, 75, 59.

(20) Wu, D.; Chandler, D.; Smit, B. J . Phys. Chem. 1992, 96, 4077. 
of the adsorption/depletion profiles of particles with one directional attractiveforce. Someattention is paid to the limit of infinitely strong attractions between the fluid particles. In this limit full association of thefluid particles into dimers occurs. In section 3 the formalism for fluids consisting of particles with twodirectional attractiveforces is presented. F or the case of strong associations between the particles, a polydisperse lattice fluid of polymeric chains near an impenetrable wall is formed.

Themethod that is used in sections 2 and 3 to introduce a wall intothefluid was developed by Henderson, Abraham and Barker ${ }^{11}$ to study theadsorption of a continuum fluid at a structureless surface. It was used by Yethiraj and Hall $^{21}$ to obtain the static adsorption characteristics of a polymericfluid at a hard wall within the RISM approach. ${ }^{22}$ The method consists of mixing a simple fluid particle $\mathrm{e}^{5}$ intotheassociating fluid, after which this particleis "blown up" in two directions to form a flat wall. It is shown in sections 2 and 3 that it is particularly easy to employ this method on thecubiclattice. In section 4 webriefly outline the Monte Carlo simulation methods used for the singly and doubly associating lattice fluids. In section 5 , the calculated adsorption profiles for the dimerizing and polymerizing fluids near hard and interacting walls are compared to the simulation data and the results are discussed. Conclusions are collected in section 6.

\section{Adsorption of Particles with One Directional Attractive Force}

In this section Wertheim's two-density formalism ${ }^{6,7}$ is adopted for the calculation of the adsorption profiles of a 3D cubic lattice fluid of singly associating particles at an impenetrablewall. Theparticles occupy exactly onelattice site and can bind into dimers due to an off-center associative particle-particle interaction. The walls are allowed to have a nonassociative interaction with the particles in the layer closest to the wall. The method ${ }^{11}$ used to introduce the wall into the fluid was devel oped to study the static adsorption behavior of continuum hard sphere fluids near an impenetrable structureless wall. For our case, its basic steps are as follows.

First, the Ornstein-Zernike matrix equation for a mixture of associating and simple (nonassociating) particles is written down. Then, the packing fraction of the simple fluid particles is reduced to zero. This assures that encounters of nonassociating simple particles are absent. After this, the simple particles are "blown up" along the $m$ - and $n$-axis of the $(1, m, n)$-coordinate system by removing the $m$ - and $n$-dependence of the correlations between thesimpleand associating particles. Theaverage correlation between the wall and an associating particle can then directly be related to the concentration of the associating particles near the wall.

The potential model used to describe the interactions between the associating particles is ${ }^{6,23}$

$$
u(1,2)=u_{R}(1,2)+u_{s_{1}, s_{2}}(1,2)
$$

where $(1,2)$ symbolically denotes the coordinates and orientations of particles 1 and 2 . In eq 1 , the repulsive part is only dependent on the distance $(I, m, n)$ between particles 1 and 2:

$$
u_{R}(0,0,0) \rightarrow \infty
$$

(21) Yethiraj, A.; Hall, C. K. J . Chem. Phys. 1991, 95, 3749.

(22) Chandler, D.; Andersen, H. C. J . Chem. Phys. 1972, 57, 1930.

(23) J anssen, R. H. C.; Nies, E.; Smit, B. Mol. Phys., submitted.

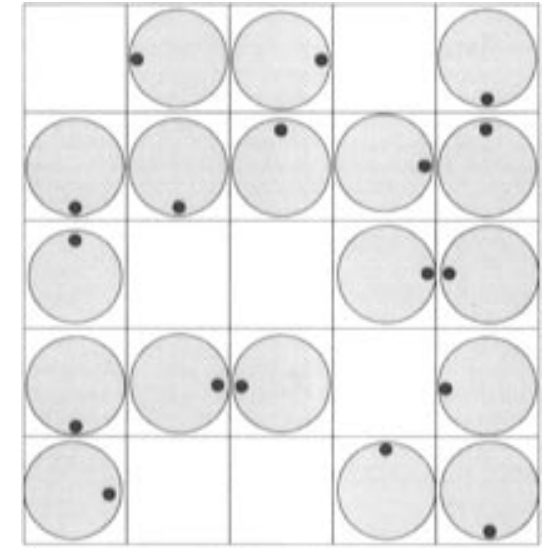

Figure 1. Fraction of an associating latticefluid in which four nearest neighbor bonds are visible.

$$
u_{R}(I, m, n)=0 \quad \text { otherwise }
$$

Note that $u_{R}(I, m, n)$ regulates that the particles occupy exactly onelatticesite. Theattractivepart of the potential $\mathrm{u}_{\mathrm{s}_{1}, \mathrm{~s}_{2}}(1,2)$ is a function of the interparticle distance $(\mathrm{I}, \mathrm{m}$, n) and of the orientations $\Omega_{1}$ and $\Omega_{2}$ of the attractivesites $\mathrm{s}_{1}$ and $\mathrm{s}_{2}$ on the particles:

$$
\begin{gathered}
\mathrm{u}_{\mathrm{s}_{1}, \mathrm{~s}_{2}}\left(0,0,0, \Omega_{1}, \Omega_{2}\right)=0 \\
\mathrm{u}_{\mathrm{s}_{1}, \mathrm{~s}_{2}}\left(\mathrm{I}, \mathrm{m}, \mathrm{n}, \Omega_{1}, \Omega_{2}\right)=\mathrm{u}_{\mathrm{attr}} \\
\text { if } \Omega_{1}=-\Omega_{2} \text { and } \mathrm{I}^{2}+\mathrm{m}^{2}+\mathrm{n}^{2}=1 \text { and } \\
\operatorname{sgn}(\mathrm{I}+\mathrm{m}+\mathrm{n})\left(\mathrm{I}^{2}+2 \mathrm{~m}^{2}+3 \mathrm{n}^{2}\right)=\Omega_{1} \\
\mathrm{u}_{\mathrm{s}_{1}, \mathrm{~s}_{2}}\left(\mathrm{l}, \mathrm{m}, \mathrm{n}, \Omega_{1}, \Omega_{2}\right)=0 \quad \text { otherwise }
\end{gathered}
$$

where an attractive site can point in the directions $+\mathrm{I}\left(\Omega_{\mathrm{i}}\right.$ $=1),-\mathrm{I}\left(\Omega_{\mathrm{i}}=-1\right),+\mathrm{m}\left(\Omega_{\mathrm{i}}=+2\right),-\mathrm{m}\left(\Omega_{\mathrm{i}}=-2\right),+\mathrm{n}\left(\Omega_{\mathrm{i}}\right.$ $=+3)$, and $-\mathrm{n}\left(\Omega_{\mathrm{i}}=-3\right)$. The conditions $\Omega_{1}=-\Omega_{2}, 1^{2}+$ $m^{2}+n^{2}=1$, and $\operatorname{sgn}(1+m+n)\left(1^{2}+2 n^{2}+3 m^{2}\right)=\Omega_{1}$ assure that bond formation only occurs between nearest neighbor particles that havetheir attractivesites oriented toward each other (see Figure 1). The interactions that involvethenonassociating simpleparticles areconsidered further on.

In the two-density formalism, the packing fraction $\eta$ of theassociating particles, which is thefraction of thelattice volume filled with associating particles, is split into the fractions of particles that have $\left(\eta_{1}\right)$ and havenot $\left(\eta_{0}\right)$ formed a bond with another associating particle ${ }^{6}$

$$
\eta=\eta_{0}+\eta_{1}
$$

The simple fluid particles do not have a directional attractive potential. Therefore, they are not involved in associative bonding and their packing fraction is not divided into parts as in eq 4. The Ornstein-Zernike matrix equation for a mixture of associating particles at packing fraction $\eta$ and simple fluid particles at $\eta_{M}$ is then given by ${ }^{6}$

$$
\mathbf{H}(1,2)=\mathbf{C}(1,2)+\frac{1}{6} \sum_{\mathbf{r}_{3,}, \Omega_{3}} \mathbf{C}(1,3) \sigma \mathbf{H}(3,2)
$$

where the argument $(1,2)$ denotes the dependence of $\mathbf{H}$ and $\mathbf{C}$ on the coordinates, i.e., position and orientation of the associating site, of particles 1 and 2 . For the mixture of associating and simple particles, the matrix $\mathbf{H}(1,2)$ is given by 


$$
\mathbf{H}(1,2)=\left|\begin{array}{lll}
h_{00} & h_{01} & h_{0} \\
h_{10} & h_{11} & h_{1} \\
h_{0} & h_{1} & h
\end{array}\right|(1,2)
$$

and the density matrix is given by

$$
\sigma=\left|\begin{array}{lll}
\mathrm{h} & \eta_{0} & 0 \\
\eta_{0} & 0 & 0 \\
0 & 0 & \eta_{\mathrm{M}}
\end{array}\right|
$$

The $(1,2)$ on the right hand side of eq 6 denotes that all el ements of $\mathbf{H}$ depend on $(1,2)$. Thematrix $\mathbf{C}(1,2)$ is of the same form as $\mathbf{H}(1,2)$. The $\mathrm{h}_{\mathrm{ij}}(1,2)$ in eq 6 denote a part of the total correlation between two associating particles, the $h_{i}(1,2)$ denote a part of the total correlation between an associating and a simple particle, and $h(1,2)$ denotes the total correlation between two simple particles. The $c_{i j}(1,2), c_{i}(1,2)$, and $c(1,2)$ denote respectively the partial direct correlations between two associating particles, an associating and a simple particle, and two simple particles. ${ }^{6}$ The elements of $\mathbf{H}(1,2)$ and $\mathbf{C}(1,2)$ depend on the orientation $\Omega_{\mathrm{s}}$ of the attractive site $\mathrm{s}$ of each associating particle that is considered

$$
\begin{gathered}
\mathrm{h}_{\mathrm{ij}}(1,2)=\mathrm{h}_{\mathrm{ij}}\left(\mathrm{I}, \mathrm{m}, \mathrm{n}, \Omega_{1}, \Omega_{2}\right) \\
\mathrm{h}_{\mathrm{i}}(1,2)=\mathrm{h}_{\mathrm{i}}\left(\mathrm{I}, \mathrm{m}, \mathrm{n}, \Omega_{1}\right)=\mathrm{h}_{\mathrm{i}}\left(\mathrm{I}, \mathrm{m}, \mathrm{n}, \Omega_{2}\right)=\mathrm{h}_{\mathrm{i}}(\mathrm{I}, \mathrm{m}, \mathrm{n}) \\
\mathrm{h}(1,2)=\mathrm{h}(\mathrm{I}, \mathrm{m}, \mathrm{n})
\end{gathered}
$$

We write $h_{\mathrm{i}}\left(I, \mathrm{~m}, \mathrm{n}, \Omega_{\mathrm{i}}\right)=\mathrm{h}_{\mathrm{i}}(I, \mathrm{~m}, \mathrm{n})$ because explicit orientational dependence only arises in case of an interaction between two associating particles. If thethird particlein eq 5 is a simple nonassociating particle, there is only a formal dependence on the orientation of this particle, and $\Sigma_{\Omega_{3}}$ can simply be replaced by the factor 6 .

B efore the closure equations necessary to calcul ate the adsorption profiles areintroduced, weperform the matrix multiplications in eq 5 and takethelimit $\eta_{\mathrm{M}} \rightarrow 0$ toexclude encounters of simple fluid particles. The result is nine equations distributed over four sets. The four sets are given by

$$
\begin{aligned}
\mathrm{h}_{\mathrm{ij}}(1,2)=\mathrm{c}_{\mathrm{ij}}(1,2)+\frac{1}{6} \sum_{\mathrm{r}_{3,}, \Omega_{3}}\left[\mathrm{c}_{\mathrm{i} 0}(1,3) \eta \mathrm{h}_{0 \mathrm{j}}(3,2)+\right. \\
\left.\mathrm{c}_{\mathrm{i0}}(1,3) \eta_{0} \mathrm{~h}_{1 \mathrm{j}}(3,2)+\mathrm{c}_{\mathrm{i} 1}(1,3) \eta_{0} \mathrm{~h}_{0 \mathrm{j}}(3,2)\right] \\
\mathrm{h}_{\mathrm{i}}(1,2)=\mathrm{c}_{\mathrm{i}}(1,2)+\frac{1}{\sigma_{\mathrm{r}_{3}, \Omega_{3}}}\left[\mathrm{c}_{\mathrm{i} 0}(1,3) \eta \mathrm{h}_{0}(3,2)+\right. \\
\left.\mathrm{c}_{\mathrm{i} 0}(1,3) \eta_{0} \mathrm{~h}_{1}(3,2)+\mathrm{c}_{\mathrm{i} 1}(1,3) \eta_{0} \mathrm{~h}_{1}(3,2)\right] \\
\mathrm{h}_{\mathrm{i}}(1,2)=\mathrm{c}_{\mathrm{i}}(1,2)+\frac{1}{6} \sum_{\mathrm{r}_{3}, \Omega_{3}}\left[\mathrm{c}_{0}(1,3) \eta \mathrm{h}_{0 \mathrm{i}}(3,2)+\right. \\
\left.\mathrm{c}_{0}(1,3) \eta_{0} \mathrm{~h}_{1 \mathrm{i}}(3,2)+\mathrm{c}_{1}(1,3) \eta_{0} \mathrm{~h}_{0 \mathrm{i}}(3,2)\right] \\
\mathrm{h}(1,2)=\mathrm{c}(1,2)+\frac{1}{6} \sum_{\mathrm{r}_{3}, \Omega_{3}}\left[\mathrm{c}_{0}(1,3) \eta \mathrm{h}_{0}(3,2)+\right. \\
\left.\mathrm{c}_{0}(1,3) \eta_{0} \mathrm{~h}_{1}(3,2)+\mathrm{c}_{1}(1,3) \eta_{0} \mathrm{~h}_{1}(3,2)\right](9)
\end{aligned}
$$

with $\{i, j\} \in\{0,1\}$. The set for $h_{i j}(1,2)$ in eq 9 is identical to the equations that were solved previously in ref 23 to obtain the bulk correlations in the dimerizing fluid. The first set in eq 9 can thus besolved independently from the other sets in eq 9 . The method to obtain the $h_{i j}(1,2)$ for fixed values of $\eta$ and $\eta_{0}$ is outlined in detail in ref 23. Note that, with $\eta_{0}$, the degree of association $\eta_{1} / \eta$ is set via eq
4. Fixation of $\left(\eta, \eta_{0}\right)$ is therefore anal ogous to the setting of $\eta$ and the strength of the associative interaction, $u_{\text {attr. }}$. The last set in eq 9 consists of a single equation for the total correlation $\mathrm{h}(1,2)$ between two simple particles. It is of no concern to us due to the limit $\eta_{\mathrm{M}} \rightarrow 0$ that we have taken. ${ }^{11}$ F urther, we only need to consider one of the two sets, the second or third in eq 9 , for the wall-associating particle correlations $h_{i}(1,2)$. Both these sets can be empl oyed, but we chooseto use the third. This set, which consists of two equations, can bewritten in an orientation independent form without approximation, becauseweare considering parts of the orientation independent distribution (see eq 8) of an associating and a simple particle. Therefore, we can write

$$
\begin{aligned}
& \mathrm{h}_{\mathrm{i}}(\mathrm{l}, \mathrm{m}, \mathrm{n})=\mathrm{c}_{\mathrm{i}}(\mathrm{l}, \mathrm{m}, \mathrm{n})+\sum_{\mathrm{I}^{\prime}, \mathrm{m}^{\prime} \mathrm{n}^{\prime}}\left(\left(\eta \mathrm{h}_{0 \mathrm{i}}\left(\mathrm{I}^{\prime}, \mathrm{m}^{\prime}, \mathrm{n}^{\prime}\right)+\right.\right. \\
& \left.\eta_{0} \mathrm{~h}_{1 \mathrm{i}}\left(\mathrm{I}^{\prime}, \mathrm{m}^{\prime}, \mathrm{n}^{\prime}\right)\right) \mathrm{c}_{0}\left(I-\mathrm{I}^{\prime}, \mathrm{n}-\mathrm{n}^{\prime}, \mathrm{m}-\mathrm{m}^{\prime}\right)+ \\
& \left.\eta_{0} \mathrm{~h}_{0 \mathrm{i}}\left(I^{\prime}, \mathrm{m}^{\prime}, \mathrm{n}^{\prime}\right) \mathrm{c}_{1}\left(I-\mathrm{I}^{\prime}, \mathrm{m}-\mathrm{m}^{\prime}, \mathrm{n}-\mathrm{n}^{\prime}\right)\right)
\end{aligned}
$$

with $i \in\{0,1\}$.

The next step is the creation of a wall from the simple fluid particles. Removing $\mathrm{m}$ - and $\mathrm{n}$-coordinates of the $h_{i}(I, m, n)$ and the $c_{i}(I, m, n)$ effectively extends the simple particle in the $\mathrm{m}$ - and $\mathrm{n}$-directions. Only the I-distance of theassociating particletothesimpleparticleis retained, and thus, the simple fluid particle behaves as a wall:

$$
\begin{aligned}
& \mathrm{h}_{\mathrm{i}}(\mathrm{I})=\mathrm{c}_{\mathrm{i}}(\mathrm{I})+\sum_{I^{\prime}, \mathrm{m}^{\prime}, \mathrm{n}^{\prime}}\left(\left(\eta \mathrm{h}_{0 \mathrm{i}}\left(\mathrm{I}^{\prime}, \mathrm{m}^{\prime}, \mathrm{n}^{\prime}\right)+\right.\right. \\
& \left.\left.\eta_{0} \mathrm{~h}_{1 \mathrm{i}}\left(\mathrm{I}^{\prime}, \mathrm{m}^{\prime}, \mathrm{n}^{\prime}\right)\right) \mathrm{c}_{0}\left(I-\mathrm{I}^{\prime}\right)+\eta_{0} \mathrm{~h}_{0 \mathrm{i}}\left(\mathrm{I}^{\prime}, \mathrm{m}^{\prime}, \mathrm{n}^{\prime}\right) \mathrm{c}_{1}\left(I-\mathrm{I}^{\prime}\right)\right)
\end{aligned}
$$

Equation 11 is the central result of this section. Note that the $h_{i j}(I, m, n)$, which are the parts of the correlation between two associating particles, are still dependent on three coordinates.

The $h_{i}(l)$, that are found from eq 11 upon combination with an appropriate closure equation, arethepartial wallassociating particle correlations that can be used to construct theconcentration profile of theassociating fluid at the wall.

We have complemented eq 11 with a Percus-Yevick type closure relation, given by

$$
y_{i}(I)=g_{i}(I)-c_{i}(I)
$$

and the exact relations

$$
g_{i}(I)=e^{-\beta u_{w}(l)} y_{i}(I)
$$

in which $u_{w}(l)$ is the symmetric $\left(u_{w}(+I)=u_{w}(-I)\right)$ wallassociative particle potential. It is given by

$$
\begin{gathered}
u_{w}(0)=+\infty \\
u_{W}( \pm 1)=u_{W} \\
u_{W}( \pm l)=0 \quad \text { otherwise }
\end{gathered}
$$

Notethat $u_{w}(l)$ is orientation independent; theorientations of the associating fluid particles are of no influence on the wall-particle interaction $u_{w}(l)$. The relations between the partial wall-fluid distribution, $g_{i}(I)$, and the partial wall-fluid correlation, $h_{i}(l)$, are ${ }^{6}$

$$
h_{i}(I)=g_{i}(I)-\delta_{i 0}
$$

Equation 15 (and eqs 12 and 13) deals with only one associating particle, which explains the single index. 
Equations 11-15 form a complete set of equations that can be solved for the $h_{i}(l)$, if we have obtained $h_{i j}(l, m, n)$ and $c_{i j}(I, m, n)$ from the first set in eq 9 with the methods outlined in ref 23 . The solution method for obtaining the $h_{i}(I)$ is outlined in theAppendix. Oncethe $h_{i}(I)$ are known, the partial wall-fluid distributions $g_{i}(I)$ are found from eq 15. The concentration profile can then be constructed from

$$
\eta g(I)=\eta g_{0}(I)+\eta_{0} g_{1}(l)
$$

From the concentration profile $\eta g(I)$, an excess adsorbed amount $\Gamma$ is defined by

$$
\Gamma(\eta)=\sum_{l=1}^{\text {lbulk }} \eta(g(I)-1)
$$

where $g(l)-1$ denotes the deviation of the associating fluid distribution at the wall from the bulk value 1 . The $I_{\text {bulk }}$ should be chosen large enough for the summation to reach into the bulk of the adsorbing fluid. An adsorption isotherm is constructed by plotting $\Gamma(\eta)$ versus $\eta$.

Note that the theory outlined in this section does not providethecorrelations between theassociating particles in the interfacial layers. It only considers wall-fluid correlations. Themethod outl ined in this section can also beused to study theadsorption of associating particles on an attracting "rod" if only one coordinate is removed in going from eq 10 to eq 11 . Results for thestaticadsorption behavior of thesingly associating fluid near an attracting wall are presented in section 5 .

\section{Adsorption of a Polymerizing Cubic Lattice Fluid}

In this section thethree-density formal ism ${ }^{8,9}$ is adapted to calculate the concentration profiles of a cubic lattice fluid consisting of particles with two directional attractive forces at an impenetrablewall. Thewall is again allowed to have a nonspecific nearest neighbor interaction with theparticlesthat occupy onelatticesiteeach. The method itself is completely analogous to the method described in the previous section. If theattractions between the doubly associating particles arerelatively strong, chain molecules will be formed. The density profiles are therefore the result of theadsor ption of a polydisperse polymericlattice fluid at an impenetrable wall.

The potential model that is used involves a summation over the two attractive sites of each particle:8

$$
u(1,2)=u_{R}(1,2)+\sum_{s_{1}=1}^{2} \sum_{s_{2}=1}^{2} u_{s_{1}, s_{2}}(1,2)
$$

The potentials $u_{R}$ and $u_{s_{1}, s_{2}}$ are defined by eqs 2 and 3 , respectively. We havechosen the sites on a particleto be indistinguishable. Kalyuzhnyi and Stell ${ }^{24}$ have generalized Wertheim's approach 8,9 to such systems, and herewe rely on their graphical analysis.

TheOZ matrix equation for a system of simple particles at packing fraction $\eta_{\mathrm{M}}$ and doubly associating particles at packing fraction $\eta$ is still given by eq 5 with the modified matrices

(24) Kalyuzhnyi, Yu. V.; Stell, G. Mol. Phys. 1993, 78, 1247.

$$
\mathbf{H}(1,2)=\left|\begin{array}{llll}
\mathrm{h}_{00} & \mathrm{~h}_{01} & \mathrm{~h}_{02} & \mathrm{~h}_{0} \\
\mathrm{~h}_{10} & \mathrm{~h}_{11} & \mathrm{~h}_{12} & \mathrm{~h}_{1} \\
\mathrm{~h}_{20} & \mathrm{~h}_{21} & \mathrm{~h}_{22} & \mathrm{~h}_{2} \\
\mathrm{~h}_{0} & \mathrm{~h}_{1} & \mathrm{~h}_{2} & \mathrm{~h}
\end{array}\right|(1,2)
$$

and

$$
\sigma=\left|\begin{array}{llll}
\sigma_{2} & \sigma_{1} & \sigma_{0} & 0 \\
\sigma_{1} & \sigma_{0} & 0 & 0 \\
\sigma_{0} & 0 & 0 & 0 \\
0 & 0 & 0 & \eta_{\mathrm{M}}
\end{array}\right|
$$

in which

$$
\sigma_{\mathrm{i}}=\sum_{\mathrm{j}=0}^{\mathrm{i}} \eta_{\mathrm{j}}
$$

with $\{\mathrm{i}, \mathrm{j}\} \in\{0,1,2\}$ and $\eta=\eta_{0}+\eta_{1}+\eta_{2}=\sigma_{2}$. The $\eta_{\mathrm{j}}$ in eq 21 denote the packing fractions of the associating particles that have formed $j \in\{0,1,2\}$ specific bonds. The matrix for $\mathbf{C}(1,2)$ is of the same form as the $\mathbf{H}(1,2)$ matrix. The $h_{i j}(1,2)$ in eq 19 denote parts of the total two-particle correl ation between two associating particles; the $h_{i}(1,2)$ concern an associating and a simple particle, and $h(1,2)$ is the total correlation between two simple particles.

Matrix multiplication of eq 5 and taking the limit $\eta_{\mathrm{M}}$ $\rightarrow 0$ results in sixteen equations distributed over four sets:

$$
\begin{gathered}
\mathrm{h}_{\mathrm{ij}}(1,2)=\mathrm{c}_{\mathrm{ij}}(1,2)+\frac{1}{6} \sum_{\mathrm{r}_{3}, \Omega_{3}}\left[\mathrm{c}_{\mathrm{i} 0}(1,3) \sigma_{2} \mathrm{~h}_{0 \mathrm{j}}(3,2)+\right. \\
\mathrm{c}_{\mathrm{i} 1}(1,3) \sigma_{1} \mathrm{~h}_{0 \mathrm{j}}(3,2)+\mathrm{c}_{\mathrm{i} 2}(1,3) \sigma_{0} \mathrm{~h}_{0 \mathrm{j}}(3,2)+ \\
\mathrm{c}_{\mathrm{i} 0}(1,3) \sigma_{1} \mathrm{~h}_{1 \mathrm{j}}(3,2)+\mathrm{c}_{\mathrm{i} 1}(1,3) \sigma_{0} \mathrm{~h}_{\mathrm{j}}(3,2)+ \\
\left.\mathrm{c}_{\mathrm{i} 0}(1,3) \sigma_{0} \mathrm{~h}_{2 \mathrm{j}}(3,2)\right] \\
\mathrm{h}_{\mathrm{i}}(1,2)=\mathrm{c}_{\mathrm{i}}(1,2)+\frac{1}{6} \sum_{\mathrm{r}_{3,}, \Omega_{3}}\left[\mathrm{c}_{\mathrm{i} 0}(1,3) \sigma_{2} \mathrm{~h}_{0}(3,2)+\right. \\
\mathrm{c}_{\mathrm{i} 1}(1,3) \sigma_{1} \mathrm{~h}_{0}(3,2)+\mathrm{c}_{\mathrm{i} 2}(1,3) \sigma_{0} \mathrm{~h}_{0}(3,2)+ \\
\left.\mathrm{c}_{\mathrm{i} 0}(1,3) \sigma_{1} \mathrm{~h}_{1}(3,2)+\mathrm{c}_{\mathrm{i} 1}(1,3) \sigma_{0} \mathrm{~h}_{1}(3,2)+\mathrm{c}_{\mathrm{i}}(1,3) \sigma_{0} \mathrm{~h}_{2}(3,2)\right] \\
\mathrm{h}_{\mathrm{i}}(1,2)=\mathrm{c}_{\mathrm{i}}(1,2)+\frac{1}{6} \sum_{\mathrm{r}_{3}, \Omega_{3}}\left[\mathrm{c}_{0}(1,3) \sigma_{2} \mathrm{~h}_{0 \mathrm{i}}(3,2)+\right. \\
\mathrm{c}_{1}(1,3) \sigma_{1} \mathrm{~h}_{0 \mathrm{i}}(3,2)+\mathrm{c}_{2}(1,3) \sigma_{0} \mathrm{~h}_{0 \mathrm{i}}(3,2)+ \\
\left.\mathrm{c}_{0}(1,3) \sigma_{1} \mathrm{~h}_{1 \mathrm{i}}(3,2)+\mathrm{c}_{1}(1,3) \sigma_{0} \mathrm{~h}_{1 \mathrm{i}}(3,2)+\mathrm{c}_{0}(1,3) \sigma_{0} \mathrm{~h}_{2 \mathrm{i}}(3,2)\right] \\
\mathrm{h}(1,2)=\mathrm{c}(1,2)+\frac{1}{6} \sum_{\mathrm{r}_{3}, \Omega_{3}}\left[\mathrm{c}_{0}(1,3) \sigma_{2} \mathrm{~h}_{0}(3,2)+\right. \\
\mathrm{c}_{1}(1,3) \sigma_{1} \mathrm{~h}_{0}(3,2)+\mathrm{c}_{2}(1,3) \sigma_{0} \mathrm{~h}_{0}(3,2)+\mathrm{c}_{0}(1,3) \sigma_{1} \mathrm{~h}_{1}(3,2)+ \\
\left.\mathrm{c}_{1}(1,3) \sigma_{0} \mathrm{~h}_{1}(3,2)+\mathrm{c}_{0}(1,3) \sigma_{0} \mathrm{~h}_{2}(3,2)\right](22)
\end{gathered}
$$

The first set in eq 22 comprises nine equations, and the second and third set each contain three equations. The fourth set contains only one equation. The set for the $h_{i j}(1,2)$ in eq 22 contains the equations that determinethe parts of the total two-particle correlations in the bulk associating fluid. It was solved in ref 18 by combining it with PY-type closures and mass balance equations. In obtaining the $h_{i j}(l, m, n)$ of the bulk fluid, it was necessary ${ }^{18,25}$ toassumetheindependence of thetwointeraction sites on the associating fluid particles and to preaverage the site-site potential $u_{s_{1}, s_{2}}$ over the orientations of the

(25) Chang, J .; Sandler, S. I. J . Chem. Phys. 1995, 102, 437. 
sites. The preaveraging is given by 18,25

$$
f_{A}(l, m, n)=\frac{1}{36} \sum_{\Omega_{s_{1}, \Omega_{s_{2}}}}\left(e^{-\beta u_{s_{1}, s_{2}}\left(1, m, n, \Omega_{\mathrm{s} 1}, \Omega_{\mathrm{s} 2}\right)}-1\right)
$$

which results in

$$
\begin{array}{ll}
\mathrm{f}_{A}(1, \mathrm{~m}, \mathrm{n})=\frac{1}{36}(a-1) & \text { if } \mathrm{I}_{2}+\mathrm{m}_{2}+\mathrm{n}_{2}=1 \\
\mathrm{f}_{\mathrm{A}}(\mathrm{l}, \mathrm{m}, \mathrm{n})=0 & \text { otherwise }
\end{array}
$$

with $a=e^{-\beta u_{a t t r}}$. The $f_{A}$, which is theattractive part of the Mayer function, ${ }^{8}$ appears in the closure and mass balance equations of thebulk fluid. The $h_{i j}(I, m, n)$ of the bulk fluid are needed in this section as an input for the calculation of the adsorption profiles. In this section, we therefore al so assume the independence of interaction sites on an associating particle and employ the preaveraging of eq 24. The last set of eq 22 is a single equation for the total correlation of two simple particles. It is of no importance tous because $\eta_{\mathrm{M}} \rightarrow 0$ guarantees that encounters of simple fluid particles do not occur. The concentration profiles of the associating fluid near the impenetrable wall can be calculated from the second or third set in eq 22. We will only consider the third set. The simple particle can be extended in the $\mathrm{m}$ - and $\mathrm{n}$-directions in the same way as outlined in the previous section. The result is

$$
\begin{gathered}
\mathrm{h}_{\mathrm{i}}(\mathrm{I})=\mathrm{c}_{\mathrm{i}}(\mathrm{I})+\sum_{\mathrm{I}^{\prime}, \mathrm{m}^{\prime}, \mathrm{n}^{\prime}}\left(\left(\sigma_{2} \mathrm{~h}_{0 \mathrm{i}}\left(\mathrm{I}^{\prime}, \mathrm{m}^{\prime}, \mathrm{n}^{\prime}\right)+\sigma_{1} \mathrm{~h}_{1 \mathrm{i}}\left(\mathrm{I}^{\prime}, \mathrm{m}^{\prime}, \mathrm{n}^{\prime}\right)+\right.\right. \\
\left.\sigma_{0} \mathrm{~h}_{2 \mathrm{i}}\left(\mathrm{I}^{\prime}, \mathrm{m}^{\prime}, \mathrm{n}^{\prime}\right)\right) \mathrm{c}_{0}\left(\mathrm{l}-\mathrm{I}^{\prime}\right)+\left(\sigma_{1} \mathrm{~h}_{0 \mathrm{i}}\left(\mathrm{I}^{\prime}, \mathrm{m}^{\prime}, \mathrm{n}^{\prime}\right)+\right. \\
\left.\left.\sigma_{0} \mathrm{~h}_{1 \mathrm{i}}\left(\mathrm{I}^{\prime}, \mathrm{m}^{\prime}, \mathrm{n}^{\prime}\right)\right) \mathrm{c}_{1}\left(\mathrm{l}-\mathrm{I}^{\prime}\right)+\sigma_{0} \mathrm{~h}_{0 \mathrm{i}}\left(I^{\prime}, \mathrm{m}^{\prime}, \mathrm{n}^{\prime}\right) \mathrm{c}_{2}\left(\mathrm{I}-\mathrm{I}^{\prime}\right)\right)
\end{gathered}
$$

Equation 25 is the central equation of this section. The $\mathrm{h}_{\mathrm{ij}}(\mathrm{l}, \mathrm{m}, \mathrm{n})$ and $\sigma_{\mathrm{i}}$ that appear in eq 25 are determined from thesolution of thebulk properties of the associating lattice fluid. ${ }^{18}$ The partial wall-associating fluid distributions $h_{i}(I)$ can now be found from eq 25 by combining it with a closure equation. We again employ the Percus-Y evicklike closure eqs 12 and 13 . The wall-associating particle interaction potential is nonspecific and still given by eq 14. The relation of $h_{i}(I)$ to the partial wall-particle distributions $g_{i}(I)$ is given by eq 15 . The index $i$ that appears in eqs $12-15$ and 25 now runs over $\{0,1,2\}$.

Equations $12-15$ and 25 constitutea set of six equations in the six unknowns $c_{0}(0), c_{0}(1), c_{1}(0), c_{1}(1), c_{2}(0)$, and $c_{2}(1)$ that is solved along the lines outlined in the Appendix. Once the unknowns are found, the $h_{i}(l)$ can directly be constructed (seetheAppendix). The concentration profile of the fluid at the impenetrable wall is finally obtained from

$$
\eta g(l)=\sum_{\mathrm{i}=0}^{2} \sigma_{\mathrm{i}} \mathrm{g}_{2-\mathrm{i}}(\mathrm{l})
$$

in which $g(I)$ is the total wall-associating particle distribution function. An adsorption isotherm can be constructed from eq 26 via eq 17.

\section{Adsorption of Associating Fluids: Monte Carlo Simulations}

The concentration profiles of the dimerizing fluid (section 2) and the polymerizing fluid (section 3) were also determined by NVT-MC simulation. Thesimulations used a box that was bounded by impenetrable walls on both sides al ong the I-axis. The box had periodic boundaries in the $\mathrm{m}$ - and $\mathrm{n}$-directions. Layers were defined as $\mathrm{mn}$-planes parallel to the impenetrable walls. Particles in the layer closest to the wall experience a wall-particle interaction. Systems with zero or an attractive wallparticle energy $u_{w}$ were considered. The concentration profiles were determined by counting the numbers of particles in each layer.

Equilibration was assured by monitoring the internal energy, E, of the fluid in the box and by checking that the obtained concentrati on profiles were symmetrical ong the I-axis. A blocking method ${ }^{26}$ was used to calculate the standard deviations of the monitored averages and to assure that the simulation recipe is able to produce uncorrelated data. Thus far, we have only determined the concentration profiles from the simulations, but in the case of the polydisperse chain fluid it might also be inter esting to study ordering, reorientation, ${ }^{27,28}$ and chain length segregation effects that are induced by the impenetrable wall.

Both types of fluids that wehaveconsidered weretested at two box filling fractions: $\eta^{\prime}=0.5$ with 1000 particles in a $20 \times 10 \times 10 \mathrm{lmn}$-box and $\eta^{\prime}=0.3$ with 1536 particles in a $20 \times 16 \times 16 \mathrm{Imn}$-box (theoverall box filling fractions are denoted as $\eta^{\prime}$ to distinguish them from the packing fraction $\eta$ of the bulk region). The walls were noninteracting or had a favorable attractive energy of $u_{w}=$ $-0.25 \mathrm{k}_{\mathrm{B}} T$ or $\mathrm{u}_{\mathrm{W}}=-0.5 \mathrm{k}_{\mathrm{B}} \mathrm{T}$ with the particles in thelayers closest to the wall. Different runs were performed with associating particle-particle interactions varying from $u_{\text {attr }}=-1.0 \mathrm{k}_{\mathrm{B}} \mathrm{T}$ to $\mathrm{u}_{\mathrm{attr}}=-7.0 \mathrm{k}_{\mathrm{B}} \mathrm{T}$ (in steps of $-0.5 \mathrm{k}_{\mathrm{B}} \mathrm{T}$ or $-1.0 \mathrm{k}_{\mathrm{B}} \mathrm{T}$ ).

The simulation recipe that was used is different for both fluid types. For the simulation of the singly associating fluid, we have used a combination of bruteforce moves and moves in which a particle was taken from a dimer and bonded to a free nonbonded monomer. The latter move is included in the recipe because there is generally no energy differ ence associated with it, leading toautomatic acceptanceand enhanced equilibration. Only when a particle is moved from or to a layer next to the wall do we have to take the wall-associating particle interaction energy intoaccount in theacceptancecriterion of this move. It samples the distribution of the particles over thelatticebut does not samplethenumber of particles bonded into a dimer. Ther efore, we also need to include a sufficient amount of brute force moves in the recipe.

A recipe was employed in which $70 \%$ brute force and $30 \%$ other moves were attempted. The averageenergy in the box and the concentration profiles were tested to be independent of the exact constitution of the recipe.

Thesimulation recipe of thepolymerizing fluid was used previ ously to obtain the structural properties of the bulk region of the fluid. ${ }^{18}$ It includes brute force moves (10\%), bond flips (10\%), double bond flips (15\%), generalized reptation moves (15\%), and biased cluster moves (50\%). Indicated between the parentheses are the percentage of attempts for thetype of move. Thepolymerizing particles interact via the model potential of 2,3 , and 18 . It is forbidden for the two sites on a particle to point in the samedirection, and the chains that areformed thus show the full excluded volume. Note that this is not the case in thetheory of section 3 , becausein solving the OZ matrix one has to assume an independence of the orientations of the attractive sites on the particles, as outlined above eq 24.

All moves used in the recipe were discussed in detail in ref 18 . The only difference is that in this work the

\footnotetext{
(26) Flyvbjerg, H.; Petersen, H. G. J . Chem. Phys. 1989, 91, 461. (27) Mansfield, K. F.; Theodorou, D. N. Macromolecules 1990, 23, 4430.

(28) Yethiraj, A. J . Chem. Phys. 1994, 101, 2489.
} 


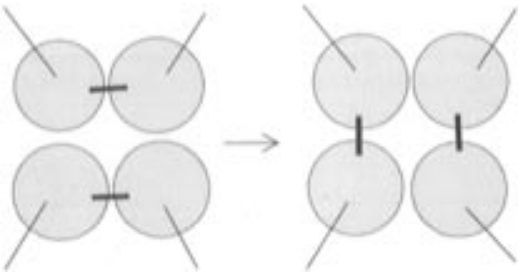

Figure 2. Illustration of a metathesis reaction.

wall-particleinteraction needs to beaccounted for in the acceptance criterion of the moves.

The brute force move consists of randomly choosing a new particle position and two orientations for the attractive sites of a randomly chosen particle. Acceptance of the move is determined by the conventional Metropolis acceptance criterion. ${ }^{17}$ The bond flip attempts to change the orientation of one of the attractive sites of a particle without moving the particle. It is meant to sample the creation/destruction of chain ends in the polymerized fluid. The third move, the double bond flip, samples the chain length distribution by imitating a metathesis reaction (see Figure 2).

This move was introduced by Olaj and Lantschbauer ${ }^{29}$ and by Mansfiel $d^{30}$ in the simulation of a polydisperse polymeric lattice fluid at full packing. The fourth move, the generalized reptation move, consists of randomly choosing two chain ends and flipping the end segment of one chain to the other chain. This move hel ps to sample the particle distribution on thelattice. Acceptance of the bond flips and the generalized reptation moves is again governed by the Metropolis acceptance criterion. N ote that there is generally no energy difference associated with the double bond flips and generalized reptation moves. The last move, the biased cluster move, was first presented in ref 18. It consists of two parts. The first part, the cluster part, ${ }^{20}$ is concerned with the selection of a part of a polymeric chain molecule: the cluster. The cluster may consist of many particles; therefore, in moving the cluster we need to alter the form of the cluster in such a way that it fits into the voids present between the other particles in the box. This is what the second part, the configurational bias scheme, ${ }^{19}$ is concerned with. Thebias that is introduced by adjusting the form of the cluster is corrected for in the acceptance criterion. ${ }^{19}$ The final scheme, $^{18}$ the bias cluster scheme, has the additional feature that it samples chain lengths. This property is most wel come in our study of polymerizing fluids in which the chains do not have fixed lengths. In ref 18 it was shown for a polymerizing bulk fluid that thebiased cluster moveis moreefficient than simple bruteforcemoves. N ote that, in thesimulation of the polymerizing fluid adsorption profiles, we need to include the wall-fluid interaction $u_{w}$ in the Rosenbluth factors. ${ }^{18,19}$

All simulations were performed in nonparallel runs on a 14 MIPS R8000 processor Silicon Graphics Power challenge $X L$ with an IRIX 6.0 operating system. Simulations for the dimerizing fluid took up to $2 \mathrm{CPU}$ hours for the strongly interacting fluids $\left(u_{a t t r}=-7.0 \mathrm{k}_{\mathrm{B}} \mathrm{T}\right)$. The polymerizing fluid simulations took up to $10 \mathrm{CPU}$ hours for $u_{a t t r}=-7.0 k_{B} T$ and 5 hours for $u_{a t t r}=-3.0 k_{B} T$. The results are available on request via e-mail at tgpken@ urc.tue.nl.

\section{Results and Discussion}

5.1. Dimerizing Fluid. In Figure 3 some concentration profiles of the singly associating lattice fluid are

(29) Olaj, O. F.; Lantschbauer, W. Makromol. Chem., Rapid Commun. 1982, 3, 847.

(30) Mansfield, M. L. J . Chem. Phys. 1982, 77, 1554.

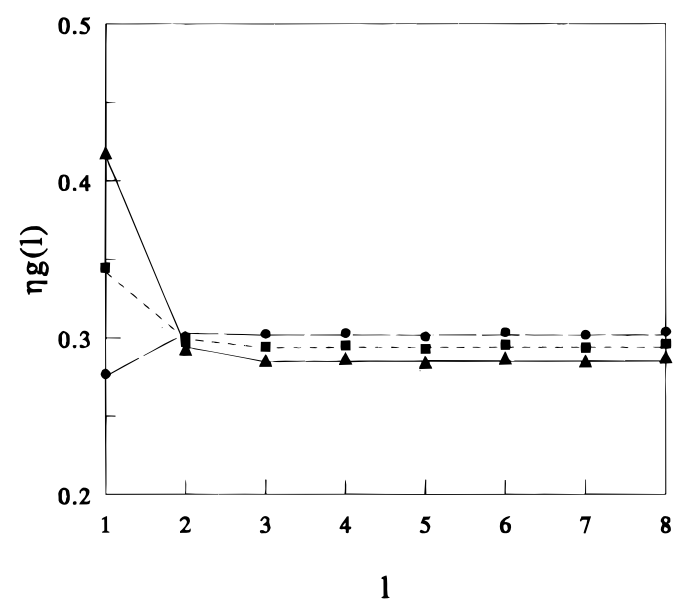

Figure 3. Concentration profiles of a singly associating fluid near a wall with $\mathrm{u}_{\mathrm{w}}=-0.5 \mathrm{k}_{\mathrm{B}} \mathrm{T}(\boldsymbol{\Delta}), \mathrm{u}_{\mathrm{W}}=-0.25 \mathrm{k}_{\mathrm{B}} \mathrm{T}(\boldsymbol{\square})$, and $\mathrm{u}_{\mathrm{W}}$ $=0.0 \mathrm{k}_{\mathrm{B}} \mathrm{T}(\bullet)$. The symbols are the results of simulations. The simulations were performed for a box filling fraction $\eta^{\prime}=0.3$ and $u_{\text {attr }}=-7.0 \mathrm{k}_{\mathrm{B}}$ T. From thesimulations we havefound $\eta$ and $\eta_{0}$ to be 0.2852 and $0.03127(\boldsymbol{\Delta}), 0.2939$ and 0.0320 (ם), and 0.3019 and $0.0320(\mathbf{0})$. With these values of $\eta$ and $\eta_{0}$ we have calculated the lines in the figureaccording to the OZ formalism of section 2.

presented. Walls are located at layer I =0 and layer I = 21. Symmetric profiles al ong the l-axis are obtai ned, but hereonly layers 1-8areshown. Threewall-fluid nearest neighbor energies were considered in Figure 3: $u_{W}=$ $0.0 \mathrm{k}_{\mathrm{B}} \mathrm{T}(\boldsymbol{\bullet}), \mathrm{u}_{\mathrm{W}}=-0.25 \mathrm{k}_{\mathrm{B}} \mathrm{T}(\boldsymbol{\square})$, and $\mathrm{u}_{\mathrm{W}}=-0.5 \mathrm{k}_{\mathrm{B}} \mathrm{T}(\boldsymbol{\Delta}) . \mathrm{In}$ thesimulations (symbols), we haveset thestrength of the particle-particleassociativeinteraction at $u_{\text {attr }}=-7.0 \mathrm{k}_{\mathrm{B}} \mathrm{T}$. This corresponds to roughly $90 \%$ of the particles being bonded into dimers. ${ }^{23}$ It is seen from thesimulations that the influence of the wall only reaches twolayers deep into thefluid, even for a relatively strong wall-fluid interaction $\mathrm{u}_{\mathrm{W}}=-0.5 \mathrm{k}_{\mathrm{B}} \mathrm{T}$ that causes a large accumulation of fluid particles in the layer closest to the wall. The depth of the profile is obviously related to the length of the main axis of the dimers: a dimer with its main axis oriented perpendicular to the wall only reaches into the second layer. In Figure 3 it is seen that the fluid tends to accumulate near the walls if the wall-fluid interaction is favorable ( $\boldsymbol{\square}$ and $\boldsymbol{\Delta}$ ). If the wall is non-interacting, a depletion is observed. Such a depletion is absent for nonassociating particles $\left(u_{\text {attr }} \rightarrow 0\right.$ ) and can be fully attributed to the entropic restrictions that the impenetrable wall imposes on the dimeric molecules that are formed if $\left|u_{\text {attr }}\right|>0$. The results of the theory of section 2 are depicted by the lines. We have drawn connecting lines through the calculated packing fractions $\eta g(l)$, to makethedistinction between thecal culated and simulated points as clear as possible. It does not mean that the theory predicts a continuous profile.

Comparisons with the theory are made at fixed bulk packing fraction $\eta$ and fixed packing fraction of nonbonded particles $\eta_{0}$. The values of $\eta$ and $\eta_{0}$ in the bulk region of the fluid (layers 4-16) are in general slightly different from the overall box values $\eta^{\prime}$ and $\eta_{0}^{\prime}$. The $\eta$ and $\eta_{0}$ that are used for comparison with thetheory are obtained from the simulations and are indicated in the caption of the figure. We have not monitored the concentration profile of $\eta_{0}$, but its bulk value was estimated from the whole box value as $\eta_{0}=\eta^{\prime} 0^{\bullet} \eta / \eta^{\prime}$. This procedure is not fully correct because it can be expected that the degree of association $1-\eta_{0} / \eta$ is a function of thelayer number $\mathrm{l}$. Theagreement between theory and simulation is neverthel ess excellent, which indicates that the wall only has a minor influence on the overall degree of association in the box. 


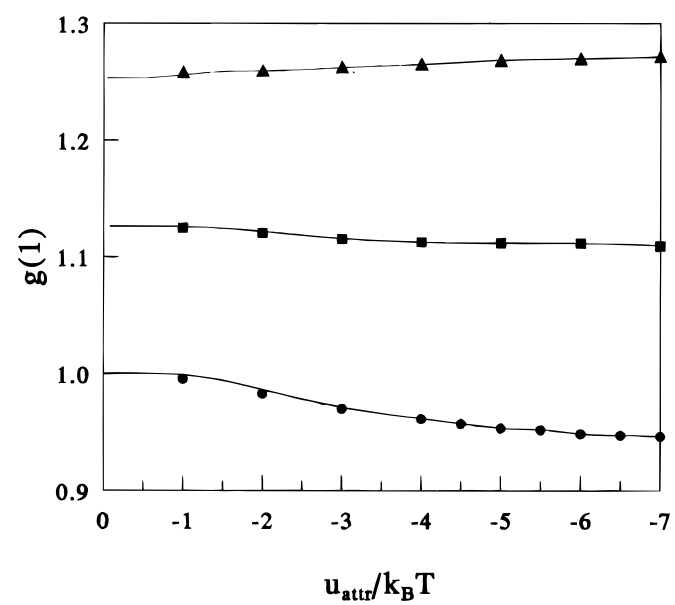

Figure 4. Simulated (symbols) and calculated (lines) adsorption in thelayer closest tothe wall for $\eta^{\prime}=0.5$ and $u_{W}=-0.5 k_{B} T$ $(\boldsymbol{\Delta}), \mathrm{u}_{\mathrm{W}}=-0.25 \mathrm{k}_{\mathrm{B}} \mathrm{T}(\boldsymbol{\square})$, and $\mathrm{u}_{\mathrm{W}}=0.0 \mathrm{k}_{\mathrm{B}} \mathrm{T}(\boldsymbol{\bullet})$ as a function of the associative interaction strength.

Note that the most appropriate comparison between the theory and the simulations would have been at fixed $\eta$ and $u_{\text {attr }}$ becauseit is thesetwo quantities that determine $\eta_{0}$. It was found previously ${ }^{23}$ that it is fully justified to make the comparison at fixed $\eta$ and $\eta_{0}$ if $\left|\mathrm{u}_{\text {attr }}\right|>3 \mathrm{k}_{\mathrm{B}} \mathrm{T}$.

Comparisons between the theory and simulations for other bulk packing fractions and degrees of association have been made and show the same excel lent agreement. Therefore, we have not depicted the full concentration profiles for such situations. An overview of theadsorption behavior at box filling fraction $\eta^{\prime}=0.5$ and $u_{w}=0.0 k_{B} T$ $(\bullet), u_{w}=-0.25 k_{B} T(\boldsymbol{\square})$, and $u_{w}=-0.5(\boldsymbol{\Delta})$ is given in Figure 4, where the amount of adsorbed material in the layer closest to the wall is plotted versus the associative interaction strength $u_{\text {attr }}(g(1)$ in Figure 4 is the packing fraction of thelayer closest to the wall divided by the bulk packing fraction). The comparison between simulation and theory is for the $\eta$ and $\eta_{0}$ observed from the simulations. Therefore, the (very small $\approx \pm 0.001$ ) numerical inaccuracies in the $\eta$ and $\eta_{0}$ obtained from the simulations also show up in the calculated curves, which explains why the curves in Figure 4 are not completely smooth.

Several interesting aspects can beobserved from Figure 4. First of all it is seen that theagreement between theory and simulation is excellent for the $u_{w}$ values that have been considered. Second, comparing the $g(1)$ values of Figure $3(g(1)=1.460)$ and Figure $4(g(1)=1.2715)$ for $\mathrm{u}_{\mathrm{W}}=-0.5 \mathrm{k}_{\mathrm{B}} \mathrm{T}$ and $\mathrm{u}_{\mathrm{attr}}=-7.0 \mathrm{k}_{\mathrm{B}} \mathrm{T}$ shows that in the case of the higher bulk packing fraction $(\eta=0.2852$ in Figure 3 and $\eta=0.4854$ in Figure 4) the tendency of the fluid to accumulate at the wall is less strong: there is a diminishing of the wall-particle correlations for higher bulk packing fractions, simply because the packing fraction at the wall can never exceed unity.

It is also seen in Figure 4 that $g(1)$ is almost fully determined by $u_{w}$ and that $u_{\text {attr }}$ is only of minor influence. This means that the favorable energetic wall-particle interaction is moreimportant than theentropicdepletion, which occurs due to ordering of the dimeric molecules at thewall. It is shown in thenext subsection that the effect of $u_{\text {attr }}$ on the concentration of the fluid near the wall is much moreinfluential for polymerizing fluids, becausein that case $u_{\text {attr }}$ has a largeeffect on the size of themolecules that are formed.

A last aspect of Figure 4 is observed by looking at the dependency of $\mathrm{g}(1)$ on $\mathrm{u}_{\text {attr }}$ for thethreelines in thefigure. Thelower curve for which $u_{w}=0$ shows a decrease of $g(1)$

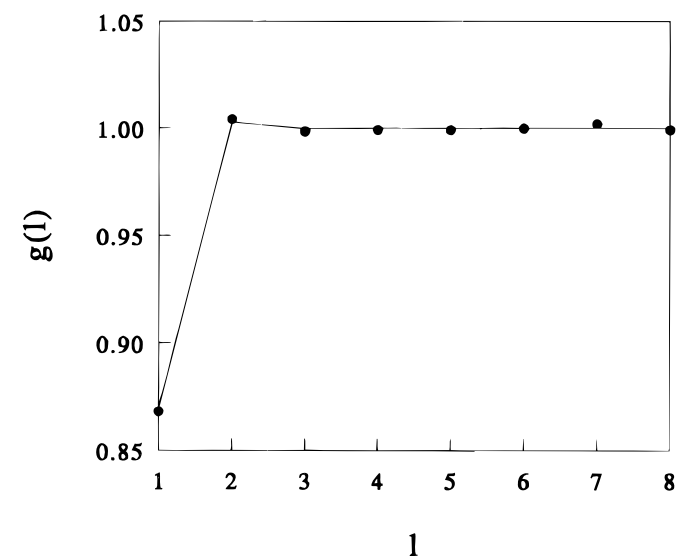

Figure 5. Adsorption profile of a dimericlatticefluid at a hard non-interacting wall for $\eta=0.1470$. Symbols areMC simulation results. The line is obtained with the method of section 2 for $u_{\text {attr }} \rightarrow-\infty$.

for higher $\left|u_{\text {attr }}\right|$ : a somewhat deeper depletion hole is observed for a more highly dimerized fluid. Thus, the entropic depletion, which is the only determining factor near a non-interacting wall, is more important for more highly dimerized systems. For the upper curve, for which $u_{W}=-0.5 k_{B} T$, the situation is reversed. Not only do the fluid particles tend to accumulate $(g(1)>1)$ at the wall, the accumulation al so increases for a stronger associative interaction $u_{\text {attr: }}$ the dimers have a stronger tendency to accumulate at the wall than the nonbonded particles, in spite of the entropic restrictions that the wall imposes on the dimers. This is because the gain in energy that accompanies the adsorption of a dimer is larger than the entropy loss. For $u_{W}=-0.25 k_{B} T$ this is not the case. Although an accumulation of particles at the wall is observed from Figure 4 for $u_{W}=-0.25 k_{B} T$, we see a slight decrease of $\mathrm{g}(1)$ for a stronger associating fluid: thewallparticleinteraction is not strong enough to counterbal ance the entropicrestriction thewall imposes on theadsorbing dimers. A decrease of $g(1)$ is therefore observed for increasing $\left|u_{\text {attr }}\right|$.

In Figure 5, the adsorption profile in the limit of infinitely strong association, $u_{\text {attr }} \rightarrow-\infty$, for which a fully dimerized lattice fluid is obtained, is drawn (full line). The symbols in Figure 5 denote points obtained with a previously outlined N PT simulation method. ${ }^{31}$ The simulation method does not attempt to break bonds between particles. This would lead to immediate rejection of the movefor thefully associated fluid $\left(\mathrm{u}_{\text {attr }} \rightarrow-\infty\right)$. Therefore, we have only sampled the distribution of the dimers over the lattice by moving them in a reptation-like fashion ${ }^{32}$ through thesimulation box. Figure 5 clearly demonstrates the accurateness of the associating fluid theory for $u_{\text {attr }} \rightarrow$ $-\infty$. I dentical results have been found at other packing fractions (up to $\eta=0.7$ ).

We conclude from the results presented in this section that the general performance of Wertheim's formalism ${ }^{6,7}$ is excellent when applied to study the adsorption profiles of singly associating cubic lattice fluids at hard and interacting impenetrable walls.

5.2. Polymerizing Fluid. In Figure 6 , some adsorption profiles of a polymerizing fluid with $\mathrm{u}_{\text {attr }}=-7.0 \mathrm{k}_{B} \mathrm{~T}$ at overall box filling fraction $\eta^{\prime}=0.3$ are depicted. The symbols indicatethesi mulation results. Thelines depict the results obtained from the theory. The bulk packing fractions that were monitored are summarized in Table 1 , together with the number average chain length $\bar{L}_{N}$ and

(31) Nies, E.; Cifra, P. Macromolecules 1994, 27, 6033.

(32) Wall, F. T.; Mandel, F. J . Chem. Phys. 1975, 63, 4592. 


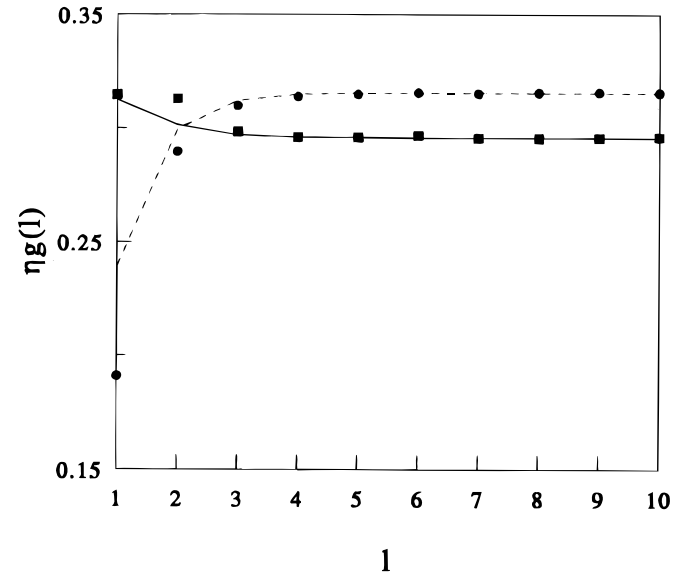

Figure 6. Adsorption profiles of a polymerizing fluid. Symbols are simulation results; lines correspond to the solution of the equations of section 3 with $u_{v}=0.0 k_{B} T$ (dashed line, $\bullet$ ) and $\mathrm{u}_{\mathrm{W}}=-0.25 \mathrm{k}_{\mathrm{B}} \mathrm{T}$ (full line, $\boldsymbol{\square}$ ). I $\mathrm{n}$ both cases, $\mathrm{u}_{\mathrm{attr}}=-0.70 \mathrm{k}_{\mathrm{B}} \mathrm{T}$ and $\eta^{\prime}=0.3$.

Table 1. Overview of Number Average Chain Lengths and Widths of Distributiona

\begin{tabular}{lllll}
\hline & symbols/lines & \multicolumn{1}{c}{$\eta$} & \multicolumn{1}{c}{$\overline{\mathrm{L}}_{\mathrm{N}}$} & $\overline{\mathrm{L}}_{\mathrm{W} / \overline{\mathrm{L}}_{\mathrm{N}}}$ \\
\hline Figure 6 & $\bullet$ & 0.3156 & 11.7 & 1.92 \\
& $\mathbf{-}$ & 0.2959 & 11.6 & 1.91 \\
& -- & 0.3156 & 12.021 & 1.917 \\
ref 18 & bulk values & 0.2959 & 11.605 & 1.938 \\
Figure 7 & $\bullet$ & 0.5113 & 11.8 & 1.92 \\
& $\mathbf{-}$ & 0.4956 & 15.6 & 1.94 \\
& -- & 0.5113 & 15.764 & 1.94 \\
ref 18 & - & 0.4956 & 15.483 & 1.937 \\
& bulk values & 0.5 & 15.5 & 1.94
\end{tabular}

a In all cases: $u_{a t t r}=-7.0 k_{B} T$.

width of the chain length distribution $\bar{L}_{w} / \bar{L}_{N}$. These were obtained from the simulations with Flory's formulas ${ }^{33}$

$$
\begin{aligned}
& \overline{\mathrm{L}}_{\mathrm{N}}=\frac{1}{1-\mathrm{p}} \\
& \frac{\overline{\mathrm{L}}_{\mathrm{W}}}{\overline{\mathrm{L}}_{\mathrm{N}}}=1+\mathrm{p}
\end{aligned}
$$

with the extent of reaction $p$ defined by ${ }^{18}$

$$
\mathrm{p}=\frac{\eta_{1}^{\prime}+2 \eta_{2}^{\prime}}{2 \eta^{\prime}}
$$

in which $\eta^{\prime}$ is the average packing fraction in the simulation box and $\eta^{\prime} ;$ are the whole box average packing fractions of particles with $i$ bonds. In eq 27, theformation of rings is neglected. ${ }^{33}$ This results in an overestimation of the actual chain length of up to $10 \%$ for the systems considered here. ${ }^{18}$ Although it is very possible to obtain the actual $\bar{L}_{N}$ and $\bar{L}_{w} / \bar{L}_{N}$ from the simulations by monitoring the full chain length distribution, ${ }^{18}$ it is not possible to obtain the full chain length distribution from thetheory of section 3. Therefore, to compare identical quantities, we also use eq 27 to analyze the simulation results.

The $\bar{L}_{N}$ and $\bar{L}_{w} / \bar{L}_{N}$ monitored from the simulations are a function of the layer number, but thus far we have not monitored the $\eta_{\mathrm{i}}$ for each layer separately. Therefore, we have only calculated whole box averages, although it is expected that there is a preference close to the wall for shorter chains when compared to longer chains: the

(33) F lory, P.J . Principles of Polymer Chemistry; Cornell University Press: Ithaca, NY, 1953.

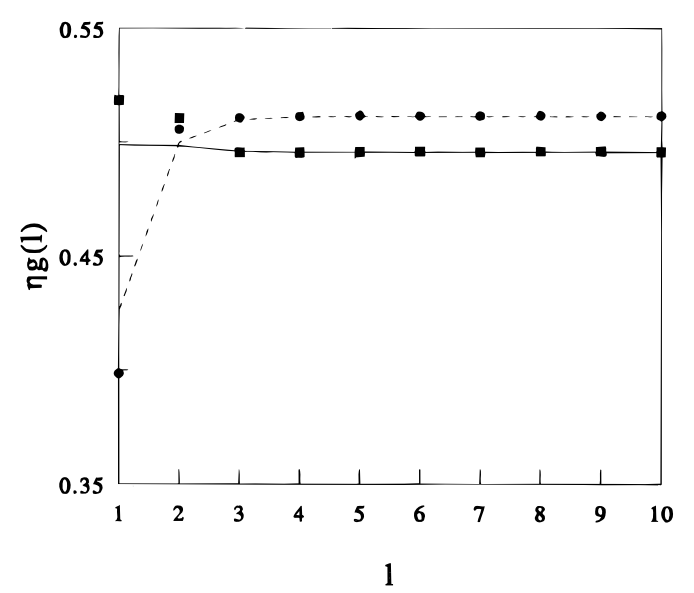

Figure 7. Caption as in Figure 6 but for $\eta^{\prime}=0.5$.

entropicrestrictions that thewall imposes on themolecules (restrictions on molecule conformation and surfaceinduced ordering of the molecules) aresmaller for shorter chains. Monitoring of the fractions of free, singly bonded, and doubly bonded particles for each layer separately would allow us to study this surface-induced segregation effect. In Figure 6, we are dealing with relatively short chains, $\bar{L}_{N} \approx 12$, for which we expect the effect to besmall. Although we have no direct proof of this, we can nevertheless see from Table 1, by comparing the whole box averages obtained in this work with theaverages obtained previously for the bulk region, ${ }^{18}$ that the surface segregation is not strong enough to causestrong deviations of the overall $20 \times 10 \times 10$ box values of $\bar{L}_{N}$ and $\bar{L}_{W} / \bar{L}_{N}$ from the values for the bulk region. This does not imply, however, that theinfluence of thewalls is unimportant in thelayers close to the wall.

Figure 7 is identical to Figure 6 but for $\eta^{\prime}=0.5$. Bulk packing fractions, number average chain lengths, and widths of the distribution are again given in Table 1 . The bulk values taken from ref 18 are also reported in Table 1. Comparison with the bul $k$ values again shows that the walls donot havea largeinfluenceon the overall averages obtained from the box.

The agreement between the calculated and simulated adsorption profiles in Figures 6 and 7 is not overall good. In the case of non-interacting walls (dashed lines) the theory seems to be qualitatively correct. It does predict a depletion hole, although the hole is not deep enough when compared to the simulation results. The origin of the depletion hole is the entropic penalty that the wall imposes on thefluid. It is not compensated by an attractive wall-fluid interaction, and therefore, a depletion of the fluid near the wall is observed.

For the interacting walls, $\mathrm{u}_{\mathrm{w}}=-0.25 \mathrm{k}_{\mathrm{B}} \mathrm{T}$ (full lines), both thetheory and thesimulations show an accumulation of particles at the wall. This accumulation is due to an energetically favorable wall-fluid interaction $u_{w}$ that compensates the entropic depletion. For $u_{w}=-0.25 k_{B} T$ (full curve) in Figure 6, the theory and the simulations both show an accumulation of particles near thewall, but we find that the theory underestimates the total amount of adsorbed material, especially in the second layer from the wall. $\mathrm{F}$ or $\mathrm{u}_{\mathrm{w}}=-0.25 \mathrm{k}_{\mathrm{B}} \mathrm{T}$ (full curve) in Figure 7 , the theory predicts an almost flat profile: the entropic depletion and energeticaccumulation are nearly balanced in the theory. The simulations, on the other hand, show a net accumulation of particles near the wall.

In both figures, the simulations for $\mathrm{u}_{\mathrm{W}}=-0.25 \mathrm{k}_{\mathrm{B}} \mathrm{T}(\boldsymbol{\square})$ show the formation of a block profile near the wall. Especially in Figure 6, the two layers closest to the wall are rather densely packed, whilethereis a sharp decrease 


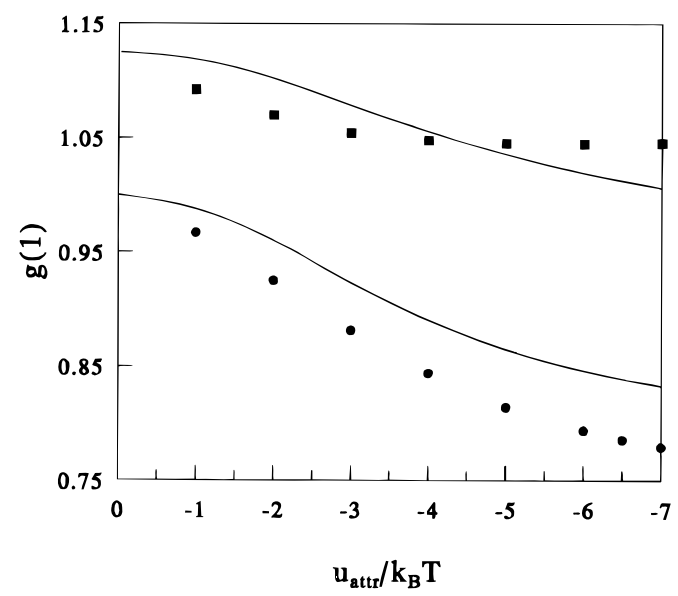

Figure 8. Normalized packing fraction, $g(1)$, in thelayer closest to the wall, versus associative interaction strength. Symbols are simulation results, and lines are calculated results: ( $\boldsymbol{\square}) \mathrm{u}_{\mathrm{W}}$ $=-0.25 \mathrm{k}_{\mathrm{B}} \mathrm{T} ;(\bullet) \mathrm{u}_{\mathrm{W}}=0.0 \mathrm{k}_{\mathrm{B}} \mathrm{T}$.

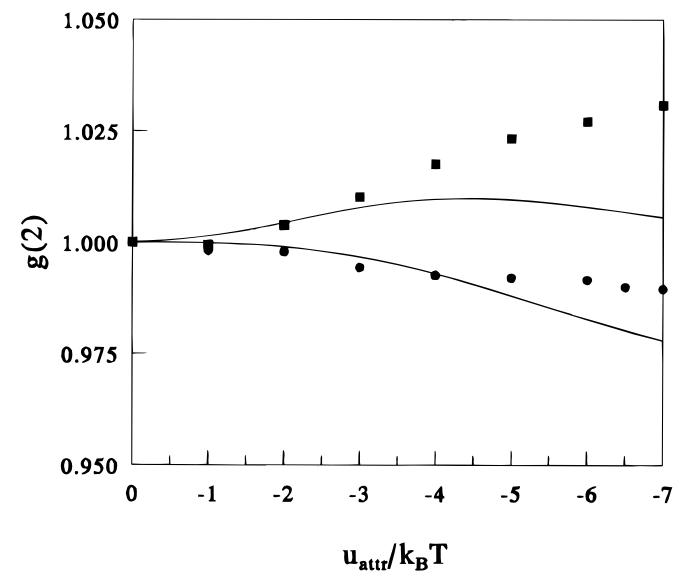

Figure 9. Caption as in Figure 8, but for the next nearest neighbor layer to the wall.

of the packing fraction in going from the second to the third layer. In Figure 7, this effect is also observed, although it is less pronounced. The block formation near the wall is obviously due to chain connectivity effects. Notethat theblock formation does not seem to becaptured by the theoretical predictions.

To obtain a clearer view on the adsorption behavior of the polymerizing lattice fluid, we have depicted an overview for box filling fraction $\eta^{\prime}=0.5$ in Figures 8 and 9 , where respectively the normalized packing fractions in the first and second layer are plotted versus the strength of theinteraction between theassociating particles. From Figure 8 it is seen that the theory and the simulations roughly have the same dependence of $g(1)$ on $u_{\text {attr. }}$. Note that although there is an accumulation $(g(1)>1$ ) of associating particles at the wall for $\mathrm{u}_{\mathrm{W}}=-0.25 \mathrm{k}_{\mathrm{B}} \mathrm{T}(\boldsymbol{\square})$, there is a decrease of the adsorption for increasing $\left|u_{a t t r}\right|$. This was also observed in Figure 4 for the adsorption of the dimerizing fluid at a wall with $\mathrm{u}_{\mathrm{w}}=-0.25 \mathrm{k}_{\mathrm{B}} \mathrm{T}$. The decrease of $g(1)$ with increasing $u_{\text {attr }}$ can be attributed to a higher entropic penalty that the wall imposes on a fluid of longer chains. It is seen from Figures 4 and 8 that the decrease of $g(1)$ with $u_{\text {attr }}$ is morepronounced for the doubly associating fluid, which was to be expected because association is much moreimportant in polymerizingfluids.

From Figure 9 it is seen that the theory is also qualitatively correct for $\mathrm{g}(2)$ if the wall is non-interacting $(\bullet)$, although the dependence of $g(2)$ on $u_{\text {attr }}$ predicted by the theory is too strong. For the interacting wall ( $\square$ ) the theory and the simulations appear to behave differently.
Thesi mulations show an increase of theadsorbed amount in thesecond layer, whereas thetheory shows a maximum. Note that the decrease of $g(1)$ ( $\mathbf{\square}$ in Figure 8) and the increase of $g(2)$ ( $\boldsymbol{\square}$ in Figure 9) for $u_{W}=-0.25 \mathrm{k}_{\mathrm{B}} \mathrm{T}$ lead to the block profiles observed in Figures 6 and 7 . It is seen in Figures 8 and 9 that, in the simulations for $u_{w}=$ $-0.25 \mathrm{k}_{\mathrm{B}} \mathrm{T}(\boldsymbol{\square})$, the decreasein $\mathrm{g}(1)$ and theincreasein $\mathrm{g}(2)$ flatten for higher $u_{\text {attr. }}$ It is most likely that for a certain $u_{\text {attr }}$ they will reach respectively a maximum and a minimum value, simply because it is improbablethat the packing fraction in the second layer will surpass the packing fraction in the first layer. For higher $\left|u_{\text {attr }}\right|$ we can certainly expect to monitor an increase of $g(3)$ with increasing $\left|u_{\text {attrr }}\right|$, although this increase will be shifted toward higher $\left|u_{\text {attr }}\right|$ when compared to that of $g(2)$. The increase of $g(3)$ corresponds totheformation of an adsorbed layer that extends deeper into the fluid if the average length of the molecules is increased.

The theory for the adsorption of a polymerizing fluid, for which some results have been presented in this subsection, is based on a precise diagrammatical analysis of the wall-fluid and fluid-fluid correlations. ${ }^{8,9}$ Despite this fact, the results are at most qualitatively correct. Reasons must lie in the approximations that were made to obtain a solvable set of equations for the bulk correlations of the polymerizing fluid. These approximations are the assumed independence of the interaction sites on each particle and the preaveraging of the site-site potential $u_{s_{1}, s_{2}}(1,2)$ over the orientations of $s_{1}$ and $s_{2}$ in the cl osure and mass balance equations for the bulk correlations. ${ }^{18,25}$ Another reason could in principle be the approximate nature of the closure equations, but this is less likely because the closures have proven to function very well in the calculation of the adsorption profiles of dimerizing fluids, as was shown in the previous subsection.

\section{Conclusions}

Cubic lattice models of singly and doubly associating fluids have been studied toassess theadsorption behavior of dimerizing and polymerizing fluids at impenetrable interacting walls. Themethod that was employed is based on Wertheim's OZ formalism ${ }^{6-9}$ and offers an elegant, relatively simple approach to study the adsorption behavior of associating lattice fluids.

Theconcentration profiles that wereobtained havebeen tested with Monte Carlo simulations. The simulation of the doubly associating lattice fluid employed a biased cluster move. ${ }^{18}$

The overall agreement between the theory and the simulations is quantitativefor thesingly associatingfluid. Theseresults thereforesuggest that Wertheim's OZ matrix formalism is also suitabletoaccurately study polymerizing fluids. Unfortunately, the results obtained for polymerizing fluids indicate that better approximations have to be adopted in solving the equations that result from Wertheim's formalism: we do not find quantitative but only qualitative agreement for the adsorption profiles at a non-interacting wall. Theadsorption of the polymerizing fluid at an interacting wall seems to be incorrectly described by the theory. The simulations show the formation of a block profile, whereas the theory shows a smoothly decreasing adsorption profile.

Finally, themethod presented hereforms an alternative tothe(polymer) RISM ${ }^{21}$ and other approaches ${ }^{12-16}$ tostudy polymer adsorption. The method does, contrary to the polymer RISM approach, not use the concept of an intramolecular distribution function to capture the conformations of the molecules near the interface, and incorporates polydispersity in an elegant way. 


\section{Appendix: Solution Scheme}

Here, weoutlinethemethod for obtaining the $h_{i}(I)$ from eqs $11-15$ for known $h_{i j}(I, m, n)$ and $c_{i j}(l, m, n)$. Solution of eq 11 is conveniently performed in Fourier space. Therefore, we apply the 1D Fourier transform

$$
\tilde{f}(u)=\sum_{1} f(I) \cos l u
$$

with inverse

$$
f(I)=\frac{1}{2 \pi} \int_{-\pi}^{\pi} \tilde{f}(u) \cos l u d u
$$

If it is assumed that the concentration profile is the same on both sides of the wall, i.e., $h_{i}(+l)=h_{i}(-1)$, we can write eq 11 as

$$
\begin{array}{r}
\tilde{h}_{i}(u)=\tilde{c}_{i}(u)+\left(\eta \hat{h}_{0 \mathrm{i}}(u, 0,0)+\eta_{0} \hat{h}_{1 \mathrm{i}}(u, 0,0)\right) \tilde{c}_{0}(u)+ \\
\eta_{0} \hat{h}_{0 \mathrm{i}}(u, 0,0) \tilde{c}_{1}(u)
\end{array}
$$

where the $\hat{h}_{i j}(u, 0,0)$ are given by 3D Fourier transform

$$
\hat{f}(u, v, w)=\sum_{l, m, n} f(l, m, n) \cos l u \cos m v \cos n w
$$

and

$f(I, m, n)=\left(\frac{1}{2 \pi}\right)^{3} \int_{-\pi}^{\pi} \int_{-\pi}^{\pi} \int_{-\pi}^{\pi} \hat{f}(u, v, w) \cos l u \cos m v x$ $\cos n w d u d v d w$ (33)

for $v=0$ and $w=0$.

The $\hat{h}_{\mathrm{ij}}(u, 0,0)$ in eq 31 can be obtained separately from the solution of the bulk equations. ${ }^{23}$ Once the $\hat{h}_{i j}(u, 0,0)$ are known, a numerically simple problem in four un- knowns $\left(c_{0}(0), c_{0}(1), c_{1}(0)\right.$, and $\left.c_{1}(1)\right)$ results. Theunknowns are found as follows.

From eq 15 and the closures, eqs 12 and 13 , we obtain the nonoverlap conditions

$$
\mathrm{h}_{\mathrm{i}}(0)=-\delta_{\mathrm{i} 0}
$$

and the contact relations

$$
c_{i}(1)=\left(h_{i}(1)+\delta_{i 0}\right)\left(1-e^{\beta u_{w}}\right)
$$

Note that the partial direct correlation functions $c_{i}(I)$ are only nonzer ofor $I \leq 1$. This is a direct consequence of the use of the Percus-Yevick-like closure, eq 12, and limits the number of unknowns to four.

The unknowns are now directly found from equations that result from combining eqs $30,31,34$, and 35

$$
\begin{gathered}
-\delta_{i 0}=\frac{1}{2 \pi} \int_{-\pi}^{\pi} \tilde{h}_{\mathrm{i}}(\mathrm{u}) \mathrm{du} \\
\frac{\mathrm{c}_{\mathrm{i}}(1)}{1-\mathrm{e}^{\beta \mathrm{uw}_{\mathrm{w}}}}-\delta_{\mathrm{i} 0}=\frac{1}{2 \pi} \int_{-\pi}^{\pi} \tilde{h}_{\mathrm{i}}(\mathrm{u}) \cos \mathrm{u} \mathrm{du}
\end{gathered}
$$

with $\tilde{c}_{i}(u)=c_{i}(0)+2 c_{i}(1) \cos u$, according to eq 29. The $c_{i}(0)$ and $c_{i}(1)$ are obtained from eq 36 with a NewtonRaphson procedure combined with a ID quadrature routine. Once the unknowns are found, we obtain the $h_{i}(I)$ from eqs 30 and 31 for $f=h_{i}$.

LA9605910 